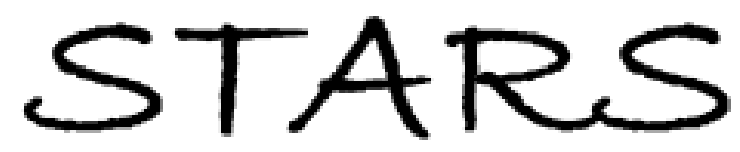

University of Central Florida

STARS

$1-1-2006$

\title{
Spatially tunable laser emission in dye-doped cholesteric polymer films
}

\author{
Yuhua Huang \\ University of Central Florida \\ Liang-Pin Chen \\ University of Central Florida \\ Charlie Doyle \\ University of Central Florida \\ Ying Zhou \\ University of Central Florida \\ Shin-Tson Wu \\ University of Central Florida
}

Find similar works at: https://stars.library.ucf.edu/facultybib2000

University of Central Florida Libraries http://library.ucf.edu

This Article is brought to you for free and open access by the Faculty Bibliography at STARS. It has been accepted for inclusion in Faculty Bibliography 2000 s by an authorized administrator of STARS. For more information, please contactSTARS@ucf.edu.

\section{Recommended Citation}

Huang, Yuhua; Chen, Liang-Pin; Doyle, Charlie; Zhou, Ying; and Wu, Shin-Tson, "Spatially tunable laser emission in dye-doped cholesteric polymer films" (2006). Faculty Bibliography 2000s. 6236.

https://stars.library.ucf.edu/facultybib2000/6236

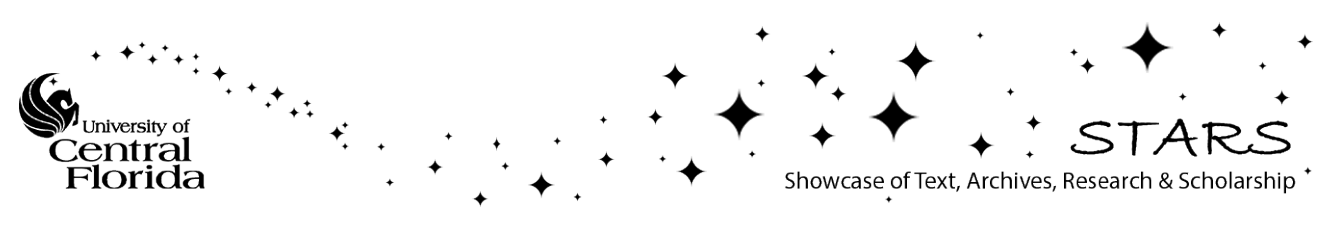




\section{Spatially tunable laser emission in dye-doped cholesteric polymer films}

Cite as: Appl. Phys. Lett. 89, 111106 (2006); https://doi.org/10.1063/1.2349826

Submitted: 05 May 2006 . Accepted: 17 July 2006 . Published Online: 12 September 2006

Yuhua Huang, Liang-Pin Chen, Charlie Doyle, Ying Zhou, and Shin-Tson Wu

\section{ARTICLES YOU MAY BE INTERESTED IN}

Cholesteric liquid crystal laser with wide tuning capability

Applied Physics Letters 86, 161120 (2005); https://doi.org/10.1063/1.1897439

Widely tunable ultraviolet-visible liquid crystal laser

Applied Physics Letters 86, 051107 (2005); https://doi.org/10.1063/1.1855405

Position sensitive, continuous wavelength tunable laser based on photopolymerizable cholesteric liquid crystals with an in-plane helix alignment

Applied Physics Letters 94, 093306 (2009); https://doi.org/10.1063/1.3089846

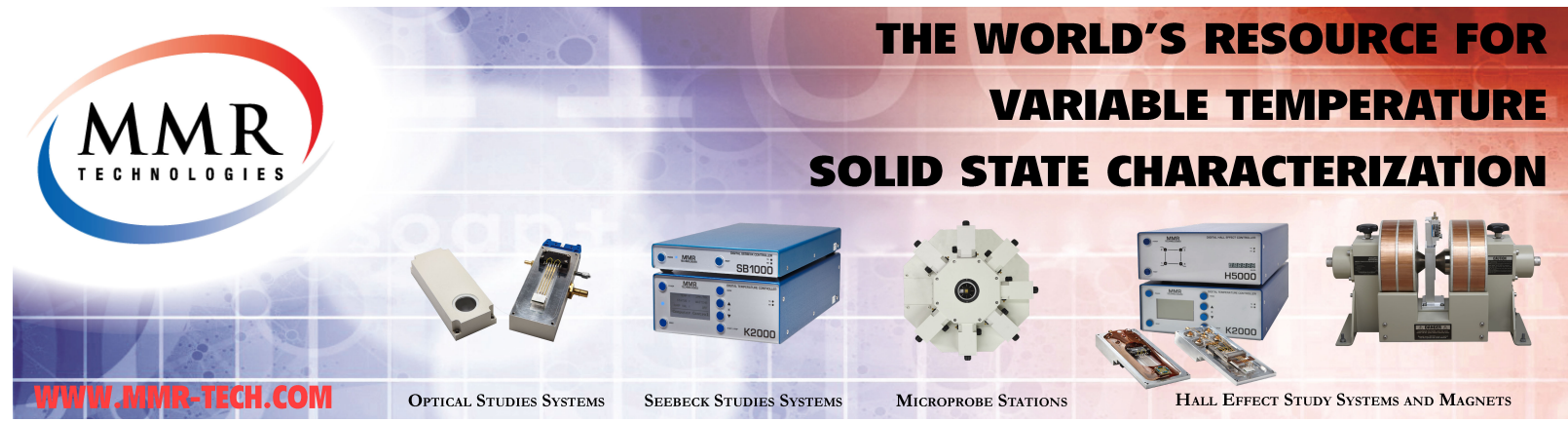




\title{
Spatially tunable laser emission in dye-doped cholesteric polymer films
}

\author{
Yuhua Huang, Liang-Pin Chen, Charlie Doyle, Ying Zhou, and Shin-Tson Wua) \\ College of Optics and Photonics, University of Central Florida, Orlando, Florida 32816
}

(Received 5 May 2006; accepted 17 July 2006; published online 12 September 2006)

\begin{abstract}
A spatially tunable laser emission of the dye-doped cholesteric polymer film is demonstrated by generating a one-dimensional gradient photonic band gap which is cured by an ultraviolet light. A frequency-doubled pulsed Nd:YAG laser is used to pump the film. The lasing wavelength is tunable from 595 to $643 \mathrm{~nm}$ by shifting the position of the film with respect to the pumping beam. Since the spatial distribution of the reflection band is fixed in the cholesteric polymer film, the tuning of the laser wavelength does not require any external field. Therefore, the film is portable and has many applications. (C) 2006 American Institute of Physics. [DOI: 10.1063/1.2349826]
\end{abstract}

Mirrorless lasers in dye-doped cholesteric liquid crystals (CLCs) have been demonstrated due to its photonic band gap property and simple fabrication process. ${ }^{1-18}$ A CLC cell is typically prepared by doping some chiral agents into a nematic LC mixture. Due to the twisting power of the chiral dopants, the LC molecules form a self-organized periodic helical structure. Since LC is a highly birefringent medium, the periodic helical structures produce a periodic modulation on the refractive index. Consequently, a one-dimensional (1D) spatially distributed photonic band gap (PBG) is established with central wavelength at $\lambda=n p$, where $p$ is the helical pitch and $n$ the average refractive index. CLCs have many unique properties such as supramolecular helicoidal periodic structure (the period can range from $100 \mathrm{~nm}$ to infinity), $100 \%$ selective reflection of a circularly polarized light, and ability to shift the selective reflection wavelength by external fields. ${ }^{1-8}$ Among these properties, the most interesting feature is that the helical pitch can be modified by means of an external field, which provides the possibility of assembling tunable mirrorless lasers. The tunable CLC lasers have been demonstrated using various methods including ultraviolet (UV) radiation, thermal effect, electric field, and generation of a spatially distributed photonic band gap. ${ }^{8-17}$

Recently, thermally and spatially tunable lasers based on the temperature dependent solubility of chiral dopants in CLC have been demonstrated. ${ }^{16,17}$ In these approaches, a temperature controller and a heating stage are required during the experiment. Once the sample is detached from a heating stage, the thermal gradient would gradually disappear. In this letter, we demonstrate a 1D spatially tunable laser by creating a dye-doped cholesteric polymer film whose gradient photonic band gap is first generated by the temperature dependent solubility of the chiral agent and then cured by an UV light. Since the 1D spatially distributed photonic band gap structure is permanently frozen in the cholesteric polymer film, no heating stage or temperature controller is needed. The laser emission wavelength can be tuned from 595 to $643 \mathrm{~nm}$ by changing the spatial position (within $\sim 20 \mathrm{~mm}$ ) of the pump beam from a frequency-doubled pulsed Nd:YAG (yttrium aluminum garnet) laser.

The chiral monomer host was prepared by mixing reactive mesogen monomer RMM154 and reactive monomer RM82 (all from Merck) at ratios of $\sim 70: 30 \mathrm{wt} \%$.

\footnotetext{
${ }^{a)}$ Electronic mail: swu@ mail.ucf.edu
}

Afterwards, we dissolved $1 \mathrm{wt} \%$ of laser dye 4-(Dicyanomethylene)-2-methyl-6-(4-dimethylaminostyryl)$4 H$-pyran (DCM) (Exciton) to the mixture. The mixture was stirred in an isotropic phase for $\sim 4 \mathrm{~h}$ to make the constituents uniformly mixed and then capillary filled into a $15 \mu \mathrm{m}$ thick LC cell in an isotropic phase. Inside the cell, both glass substrates were coated with a thin polyimide alignment layer. The antiparallel rubbing-induced pretilt angle is $\sim 3^{\circ}$. After the temperature was gradually cooled down to the room temperature $\left(\sim 23{ }^{\circ} \mathrm{C}\right)$, a chiral monomer sample with righthanded helix was formed.

In experiment, we first characterized the temperature dependent photonic band gap spectrum of the chiral monomer sample by changing the temperature from 23 to $70{ }^{\circ} \mathrm{C}$. The temperature of the dye-doped chiral monomer cell was monitored by a temperature controller. Figure 1(a) shows the transmission spectra of the chiral monomer cell at different temperatures. As the temperature increases, the PBGs of the chiral monomer sample shift toward blue wavelength and the band gap becomes narrower. This is attributed to the increased solubility of the chiral dopants in the monomers as the temperature increases. The increasingly dissolved chiral concentration in the monomers enhances the twisting power which consequently decreases the pitch length of the PBGs. In the meantime, the birefringence $(\Delta n)$ of the monomer decreases as the temperature increases. ${ }^{18}$ Since the cholesteric reflection bandwidth is proportional to $p \Delta n$, the photonic band gap becomes narrower at a higher temperature. Figure 1(b) depicts the temperature dependent central wavelength $\left(\lambda_{0}\right)$ of the PBGs. The central wavelength is defined as $\lambda_{0}$ $=\left(\lambda_{\text {long }}+\lambda_{\text {short }}\right) / 2$, where $\lambda_{\text {long }}$ and $\lambda_{\text {short }}$ stand for the wavelengths at the long and short reflection band edges, respectively. Both $\lambda_{\text {long }}$ and $\lambda_{\text {short }}$ are obtained from the measured transmission spectra of the chiral monomer sample at each temperature.

Based on the temperature dependent optical properties of the chiral monomer sample, we can easily generate a 1D spatially tunable PBG in the sample by generating a temperature gradient across the cell. To achieve this goal, we simply placed one side of the chiral monomer cell on a heating stage and left the other side in the air. By raising the temperature of the heating stage to $70{ }^{\circ} \mathrm{C}$, a $1 \mathrm{D}$ temperature gradient was formed. Since the PBG of the chiral monomer is temperature dependent, a 1D spatially distributed PBG was formed in the cell. Afterwards, we illuminated the sample with an UV light 

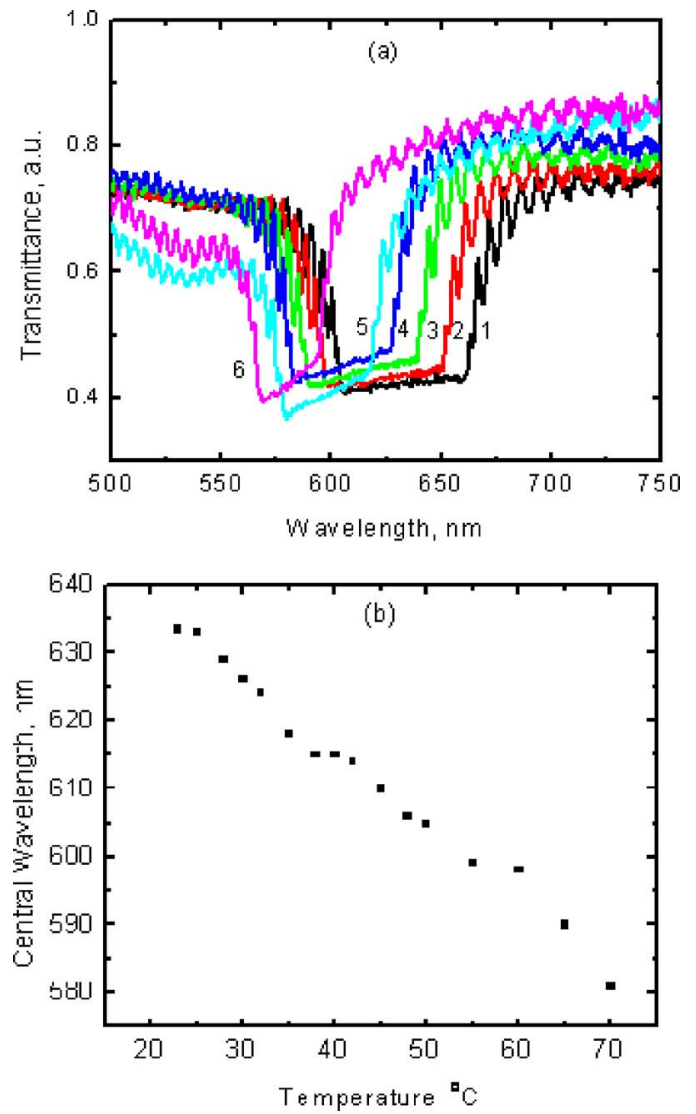

FIG. 1. (Color online) (a) PBG of the chiral monomer sample at different temperatures. Curves $1-6$ correspond to $T=23,30,40,50,60$, and $70{ }^{\circ} \mathrm{C}$, respectively. (b) The measured average reflection wavelength of the chiral monomer cell as a function of the temperature. Cell gap $d=15 \mu \mathrm{m}$.

to polymerize the monomer. A cholesteric polymer film with 1D spatially tunable photonic band gap was formed. Figure 2 shows a photo of a cholesteric polymer film after UV curing. The reflected colors spread from green (the side closer to the heating stage) to orange. In principle, we could peel off the glass substrates and obtain a $15 \mu \mathrm{m}$ polymeric film, but in our experiment we did not do so.

By doping 1 wt \% laser dyes in the chiral monomer mixture, we fabricated a dye-doped cholesteric polymer film for spatially tunable laser applications. To test the laser emission

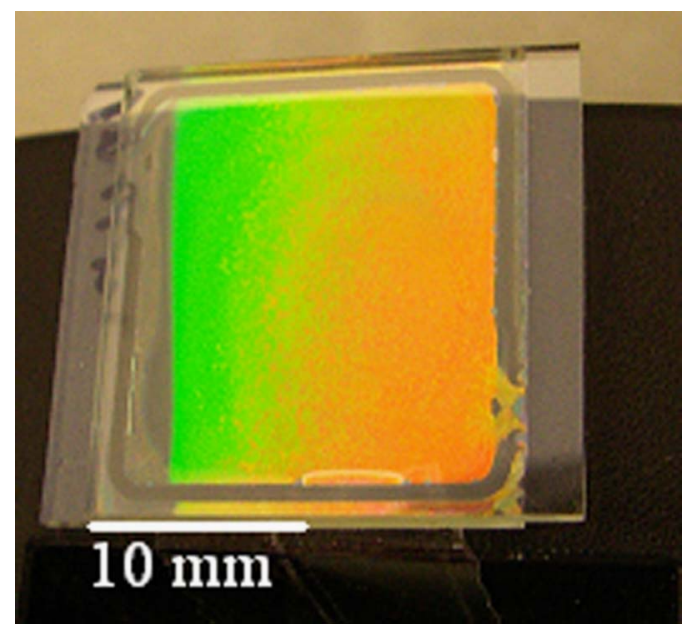

FIG. 2. (Color online) Photo of the cholesteric polymer film sample with gradient reflection band.
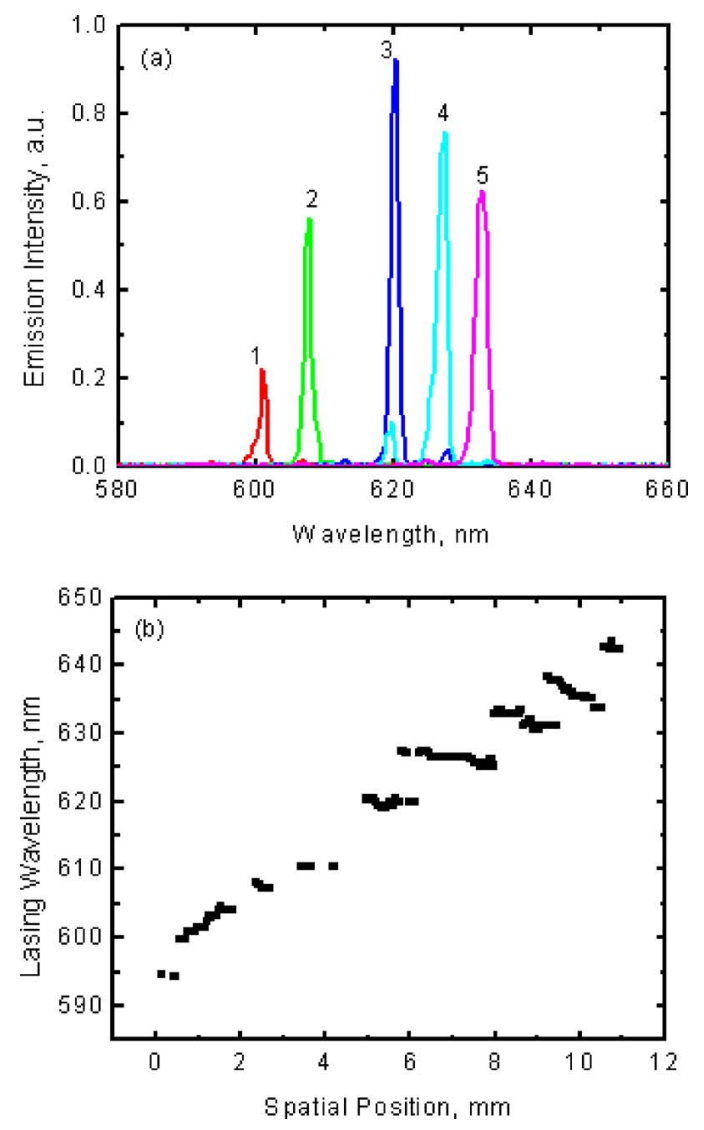

FIG. 3. (Color online) (a) Laser emission of the dye-doped cholesteric polymer film at different sample positions. Curves 1-5 correspond to the positions at $0.6,2.3,4.9,6.2$, and $8.2 \mathrm{~mm}$ relative to the starting point, respectively. (b) The lasing peak wavelength as a function of the spatial position.

properties, a second-harmonic $Q$-switched Nd:YAG pulsed laser (from Continuum, $\lambda=532 \mathrm{~nm}$ ) with vertical linear polarization was used to pump the dye-doped cholesteric polymer film. The experimental setup is similar to that described in Ref. 16. The pulse width is $4 \mathrm{~ns}$ and the repetition rate is kept at $1 \mathrm{~Hz}$ in order to reduce the heat accumulation in the sample. A beam splitter was used to divide the incoming laser into two beams: one was sent to a laser energy meter (Ophir) for monitoring the pumping pulse energy and the other was used as the excitation beam. A linear polarizer and a quarter-wave plate were used to convert the linearly polarized light into left-handed circularly polarized light to avoid the reflection by the cholesteric polymer film. A lens with $15 \mathrm{~cm}$ focal length focused the incident beam to a small spot of $\sim 160 \mu \mathrm{m}$ diameter at the sample. The output laser emission in the forward direction of the sample was collected by a lens to a fiber-optics-based universal serial bus (USB) spectrometer (0.4 nm resolution; USB HR2000, Ocean Optics).

Figure 3(a) is the plot of the laser emission spectra from the dye-doped cholesteric polymer film at different positions. To avoid the crowdedness of the figure, we plot just five lasing wavelengths. Here, the starting point $(0 \mathrm{~mm})$ is defined as the spot on the side near the heating stage. The other spatial positions are relative to the starting point. In this experiment, the pump energy was set at $100 \mu \mathrm{J} /$ pulse. However, the laser emission intensity is wavelength dependent. The highest lasing intensity occurs at $\lambda \sim 620 \mathrm{~nm}$, rather than at the dye's peak fluorescent wavelength $(\lambda \sim 608 \mathrm{~nm})$. 


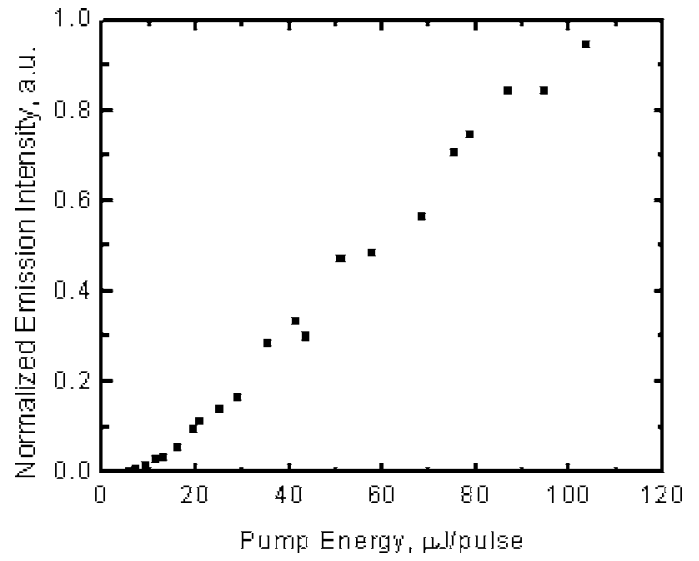

FIG. 4. Laser emission intensity from the dye-doped cholesteric polymer film as a function of the excitation energy of the pumping beam at $\lambda$ $=532 \mathrm{~nm}$.

This phenomenon results from the competition between the optical loss and gain. Generally, the laser emission can be generated through feedback amplification only when the optical gain is larger than the loss in the medium. In the cholesteric polymer film, the amplification of the optical gain is provided by the internal distributed feedback of the PBG. The laser emission can be obtained when the amplification of the optical gain traveling in the cholesteric polymer film is sufficient to overcome the loss in the medium. A higher optical gain can generate a more efficient laser emission. Usually, the optical gain spectrum is determined by the fluorescence spectrum of the dye. Since the DCM dye exhibits a maximum fluorescence at $\lambda \sim 608 \mathrm{~nm}$, the highest lasing efficiency is expected to occur at this wavelength. However, the obtained highest lasing efficiency occurs at $\lambda \sim 620 \mathrm{~nm}$. This is because the loss at $\lambda \sim 608 \mathrm{~nm}$ is larger than that at $\lambda \sim 620 \mathrm{~nm}$, as will be explained later.

Figure 3(b) shows the trend of the laser peak wavelength with respect to the spatial position. The laser emission peak shifts noticeably according to the spatial position of the cholesteric polymer film. The lasing wavelength shifts from $\lambda$ $=595 \mathrm{~nm}$ (heater side) to $643 \mathrm{~nm}$. We also noticed that the tuning of the laser wavelength with respect to the spatial position is not continuous; instead, it jumps discontinuously and hops around each lasing wavelength within a certain spatial range. The detailed mechanism for such a discontinuity is not yet completely understood but is speculated to originate from the strong anchoring effect of the rubbed surfaces.

Ideally, the PBG should shift continuously as the effective twisting capability changes continuously along the spatial position. However, because of the strong anchoring effect the polymer chain near the boundary surfaces tends to follow the rubbing directions of the substrates. A perfect PBG with sharp band edge, as shown in Fig. 1, can be formed only when the cell gap over the pitch number is equal to $m / 2$, where $m$ is an integer. Otherwise, the pitch length would have some small variations, i.e., inhomogeneous distribution along the helix in order to satisfy the boundary conditions. As a result, the band edge of the PBG would not be so sharp. The imperfect PBG dramatically decreases the density of states which, in turn, increases the optical loss. If the optical loss exceeds the gain because of the imperfect PBG, laser action would stop. Consequently, the lasing wavelength jumps within a certain spatial range, as shown in Fig. 3(b). This mechanism also explains why the peak lasing efficiency does not occur at $\lambda \sim 608 \mathrm{~nm}$.

In addition, we also investigated the laser emission intensity as a function of the excitation energy. Here, we just plot the results of the lasing wavelength at $\lambda \sim 620 \mathrm{~nm}$ as an example to show the trend of the lasing intensity with respect to the pump energy. As Fig. 4 shows, when the excitation energy exceeds a threshold, the emission intensity increases linearly with the increased pumping energy.

In conclusion, we have demonstrated a spatially tunable laser emission by generating a $1 \mathrm{D}$ spatially distributed PBG in a dye-doped cholesteric polymer film. The lasing wavelength is tunable from 595 to $643 \mathrm{~nm}$ by changing the spatial position of the dye-doped cholesteric polymer film. Since the spatially distributed reflection band is fixed in the cholesteric polymer film, the tuning of the laser wavelength does not require an electric field or temperature controller. Moreover, the film is lightweight and portable. Its widespread applications are foreseeable.

${ }^{1}$ S. Furumi, S. Yokoyama, A. Otomo, and S. Mashiko, Appl. Phys. Lett. 82, 16 (2003).

${ }^{2}$ T. Matsui, R. Ozaki, K. Funamoto, M. Ozaki, and K. Yoshino, Appl. Phys. Lett. 81, 3741 (2002).

${ }^{3}$ A. F. Munoz, P. Palffy-Muhoray, and B. Taheri, Opt. Lett. 26, 804 (2001).

${ }^{4}$ E. Alvarez, M. He, A. F. Munoz, R. Palffy-Muhoray, S. V. Serak, B. Taheri, and R. Twieg, Mol. Cryst. Liq. Cryst. Sci. Technol., Sect. A 369, 75 (2001).

${ }^{5}$ A. Chanishvili, G. Chilaya, G. Petriashvili, R. Barberi, R. Bartolino, G. Cipparrone, A. Mazzulla, and L. Oriol, Appl. Phys. Lett. 83, 5353 (2003).

${ }^{6}$ S. M. Morris, A. D. Ford, M. N. Pivnenko, and H. J. Coles, J. Appl. Phys. 97, 023103 (2005).

${ }^{7}$ Y. D. Ma and B. G. Wu, U.S. Patent No. 5,949,513 (7 September 1999).

${ }^{8}$ L. C. Chien, U. Muller, M. F. Nabor, and J. W. Doane, SID Int. Symp. Digest Tech. Papers 26, 169 (1995).

${ }^{9}$ S. Furumi, S. Yokoyama, A. Otomo, and S. Mashiko, Appl. Phys. Lett. 84, 2491 (2004)

${ }^{10}$ T. H. Lin, Y. J. Chen, C. H. Wu, A. Y. G. Fuh, J. H. Liu, and P. C. Yang, Appl. Phys. Lett. 86, 161120 (2005).

${ }^{11}$ K. Funamoto, M. Ozaki, and K. Yoshino, Jpn. J. Appl. Phys., Part 2 42, L1523 (2003).

${ }^{12}$ A. Chanishvili, G. Chilaya, G. Petriashvili, R. Barberi, R. Bartolino, G. Cipparrone, A. Mazzulla, and L. Oriol, Adv. Mater. (Weinheim, Ger.) 16, 791 (2004).

${ }^{13}$ A. Chanishvili, G. Chilaya, G. Petriashvili, R. Barberi, R. Bartolino, G. Cipparrone, A. Mazzulla, R. Gimenez, L. Oriol, and M. Pinol, Appl. Phys. Lett. 86, 051107 (2005).

${ }^{14}$ H. Yu, B. Y. Tang, J. Li, and L. Li, Opt. Express 13, 7243 (2005).

${ }^{15}$ P. V. Shibaev, R. L. Sanford, D. Chiappetta, V. Milner, A. Genack, and A. Bobrovsky, Opt. Express 13, 2358 (2005).

${ }^{16}$ Y. Huang, Y. Zhou, and S. T. Wu, Appl. Phys. Lett. 88, 011107 (2006).

${ }^{17}$ Y. Huang, Y. Zhou, C. Doyle, and S. T. Wu, Opt. Express 14, 1236 (2006).

${ }^{18}$ S. T. Wu, Phys. Rev. A 33, 1270 (1986). 